\title{
SOX10 is a novel marker of acinus and intercalated duct differentiation in salivary gland tumors: a clue to the histogenesis for tumor diagnosis
}

Rie Ohtomo ${ }^{1,7}$, Taisuke Mori ${ }^{2,7}$, Shinsuke Shibata ${ }^{3}$, Koji Tsuta ${ }^{1}$, Akiko M Maeshima ${ }^{1}$, Chihiro Akazawa ${ }^{4}$, Yukio Watabe ${ }^{5}$, Kazufumi Honda ${ }^{5}$, Tesshi Yamada ${ }^{5}$, Seiichi Yoshimoto ${ }^{6}$, Masao Asai ${ }^{6}$, Hideyuki Okano ${ }^{3}$, Yae Kanai ${ }^{2}$ and Hitoshi Tsuda ${ }^{1}$

${ }^{1}$ Department of Pathology and Clinical Laboratory, National Cancer Center Hospital, Tokyo, Japan; ${ }^{2}$ Division of Molecular Pathology, National Cancer Center Research Institute, Tokyo, Japan; ${ }^{3}$ Department of Physiology, Keio University School of Medicine, Tokyo, Japan; ${ }^{4}$ Department of Biochemistry and Biophysics, Graduate School of Health and Sciences, Tokyo Medical and Dental University, Tokyo, Japan; ${ }^{5}$ Division of Chemotherapy and Clinical Research, National Cancer Center Research Institute, Tokyo, Japan and ${ }^{6}$ Department of Head and Neck Oncology and Plastic Surgery, National Cancer Center Hospital, Tokyo, Japan

Salivary gland tumors are relatively rare and morphologically diverse and heterogeneous tumors; therefore, histogenesis-based tumor markers are sorely needed to aid in diagnosing and determining the cell type of origin. SRY-related HMG-box 10 (SOX10) protein is a transcription factor known to be crucial in the specification of the neural crest and maintenance of Schwann cells and melanocytes. In addition, positive expression has also been implicated in the major salivary gland. Here, we examined SOX10 expression in various salivary gland tumors to correlate this expression with myoepithelial markers. Overall, 76 malignant and 14 benign tumors were examined. SOX10 expression clearly delineated two distinct subtypes of human salivary gland tumors; acinic cell carcinomas, adenoid cystic carcinomas, epithelial-myoepithelial carcinomas, myoepithelial carcinomas, and pleomorphic adenomas, including the pleomorphic adenoma component of carcinoma, were SoX10 positive, while salivary duct carcinomas, mucoepidermoid carcinomas, an oncocytic carcinoma, Oncocytomas, and Warthin tumors were SOX10 negative. Also, SoX10 was expressed in solid-type or nonspecific morphology salivary gland tumors, but was not expressed in poorly differentiated squamous cell carcinomas. In normal human salivary gland tissue, SOX10 expression was specific to the nuclei of acini and both luminal and abluminal cells of intercalated ducts but not in other sites. Moreover, the murine model suggested that SoX10 continued to be expressed from the developmental stage to adulthood in the acinar and both luminal and abluminal intercalated ducts in the major salivary gland. Thus, SOX10 is a novel marker for diagnosing and understanding the histogenesis of salivary gland tumors.

Modern Pathology (2013) 26, 1041-1050; doi:10.1038/modpathol.2013.54; published online 5 April 2013

Keywords: p63; SOX10; salivary gland tumor; salivary gland development

Salivary gland tumors are relatively rare and morphologically diverse tumors with a wide variety of prognostically important histological subtypes. Although pathologists have investigated this diverse

Correspondence: Dr T Mori, DMD, PhD, Division of Molecular Pathology, National Cancer Center Research Institute, 5-1-1 Tsukiji, Chuou, Tokyo 104-0045, Japan.

E-mail: tamori@ncc.go.jp

${ }^{7}$ These authors contributed equally to this work.

Received 15 October 2012; revised 8 February 2013; accepted 9

February 2013; published online 5 April 2013 group of tumors, their diagnosis and treatment remain a complex and challenging problem. Markers that aid in diagnosing and determining the cell type of origin are greatly needed for this heterogeneous group of tumors.

A candidate for such a marker is SOX10. This transcription factor is known to be crucial in the specification and maturation of the neural crest and maintenance of Schwann cells and melanocytes. ${ }^{1}$ The immunohistochemical value of SOX10 has been described as a more sensitive and specific marker than S100 protein for melanocytic and Schwannian 
tumors. ${ }^{2}$ In addition to melanocytic and Schwannian tumors, positive expression has also been noted in myoepithelial cells of the salivary gland, bronchial submucosa, and mammary glands, in addition to case reports of SOX10 expression in two instances of parotid gland myoepithelioma. ${ }^{2}$ An interesting hypothesis explaining SOX10's mechanistic role in myoepithelial cells is that its downregulation induces the transdifferentiation of Schwann cells to myofibroblasts. ${ }^{3}$

From the standpoint of embryology, previous work has established that parotid glands originate from the oral ectoderm; ${ }^{4}$ however, the embryologic origins of the submandibular and sublingual gland epithelium have not been clearly defined, although ectodermal origins are suggested. ${ }^{4}$ In the development of mouse submandibular glands, SOX10 expression has been associated with the presence of epithelial stem/progenitor cells from embryonic day13.5 (E13.5) to adulthood. ${ }^{5}$ In addition, SOX10 has been noted to be expressed more frequently in buds (acini) than in ducts with high levels of endogenous expression in the luminal (epithelial) cells of acinic E14.5 embryos; ${ }^{6}$ however, in human salivary glands, SOX10 expression remains unclear. To our knowledge, no studies have clearly shown SOX10 expression patterns in major salivary glands and their tumors.

The objective of this study was to find whether SOX10 has the potential to serve as a pathological marker in benign and malignant salivary gland tumors and to study its expression in murine development and human major salivary gland tissues.

\section{Materials and methods}

\section{Tissue Samples}

We collected formalin-fixed paraffin-embedded specimens from 14 normal major salivary glands (8 parotid glands and 6 submandibular glands), 90 salivary tumor tissues, and 10 head and neck squamous cell carcinomas and corresponding noncancerous squamous epithelium tissues surgically resected between 1998 and 2010 at the National Cancer Center Hospital in Tokyo. All specimens were reviewed in our institution, and the histological types of the tumors were classified according to the WHO criteria. ${ }^{7}$ These tumors comprised 76 malignant tumors and 14 benign tumors: 23 adenoid cystic carcinoma, 15 carcinoma not otherwise specified, 11 carcinoma ex pleomorphic adenoma, 8 acinic cell carcinoma, 6 salivary duct carcinoma, 6 mucoepidermoid carcinoma, 6 pleomorphic adenoma, 5 epithelial-myoepithelial carcinoma, 4 myoepithelioma, 2 oncocytoma, 2 Warthin tumors, and 1 case each of Myoepithelial carcinoma and Oncocytic carcinoma. Informed consent was obtained from the patients, and the protocol of this study was reviewed by the Ethics Committee of the National Cancer Center Hospital,
Tokyo, Japan, which approved the procedures (Approval no. 2010-075).

\section{Tissue Microarray}

We selected formalin-fixed paraffin-embedded tissue blocks containing the main tumor areas and the normal salivary gland area that had been used for histological examination. Two core specimens, $2.0 \mathrm{~mm}$ in diameter, for each case were taken from these blocks and transferred to recipient blocks using a TMA Master tissue microarray (3DHistech, Budapest, Hungary). ${ }^{8}$

\section{Immunohistochemical Analysis}

TMA blocks were cut into 4- $\mu$ m-thick sections. Deparaffinized sections were subjected to hematoxylin and eosin in addition to immunohistochemical staining. Immunohistochemical staining was performed with the followed primary antibodies: SOX10 (1:200; Santa Cruz Biotechnology; N-20), p63 (1:400; Dako, Carpinteria, CA; A4A), Smooth muscle actin (1:100; Dako, 1A4), Calponin (1:200; Dako, CALP), S100 (1:1000; Dako, Rabbit polyclonal), CK14 (1:50; Novacastra; LL002), CK5/6 (1:400; DAKO; D5/16B4), and Glial fibrillary acidic protein (1:1000; Dako, Rabbit polyclonal). Each section was exposed to $0.3 \%$ hydrogen peroxide for $15 \mathrm{~min}$ to block endogenous peroxidase activity. For staining, we used an automated stainer (Dako) according to the vendor's protocol. ChemMate EnVision (Dako) methods were used for detection. Appropriate positive and negative controls were used for each antibody. Nuclear staining of $>10 \%$ tumor cells was considered positive for SOX10 and p63; cytoplasmic staining for Smooth muscle actin, calponin, CK14, CK5/6 and Glial fibrillary acidic protein; both nuclear and cytoplasmic staining for S100.

\section{Immunofluorescence Double Staining}

To determine SOX10 distribution compared with p63 in the human salivary gland, we collected five normal major salivary gland tissues blocks, which were in Tisuue microarrays. After heat-induced epitope retrieval, these five sections were incubated with primary antibodies against SOX10 (1:200; Santa Cruz Biotechnology; N-20) and p63 (1:400; Dako, A4A), followed by labeling with Alexa Fluor 488-conjugated donkey anti-goat IgG (H+L) (1:100; Molecular Probes) and Alexa Fluor 594-conjugated donkey anti-mouse IgG $(\mathrm{H}+\mathrm{L})$ (1:250; Molecular Probes), respectively. Images were collected with a confocal microscope system (LSM 510 META; Zeiss). ${ }^{9,10}$

\section{SOX10-Venus BAC Transgenic Mice}

SOX10-Venus bacterial artificial chromosome (BAC) transgenic mice have been described previously. ${ }^{11}$ 
The concept of this mouse is that SOX10-expressing cells are clearly visualized by Venus protein. The mouse sections were supplied by the Department of Physiology, Keio University School of Medicine. Briefly, E11.5, $n=2$; E12.5, $n=6$; E14.5, $n=4$; E15.5, $n=3$; E16.5, $n=4$; E17.5, $n=3$; postnatal day 0 (P0), $n=4$; and adult salivary glands, $n=4$; were fixed with $4 \%$ paraformaldehyde, and $16-\mu$ m-thick cryosections were prepared with a cryostat, CM3000 (Leica, Germany). After heat-induced epitope retrieval with Target Retrieval Solution (Dako) and autoclaving for $5 \mathrm{~min}$ at $105{ }^{\circ} \mathrm{C}$, these sections were incubated with primary antibodies goat anti-SOX10 (1:200; R\&D Systems), Alexa Fluor 488-conjugated rabbit anti-GFP (Invitrogen), goat anti-GFP antibody (1:200; Rockland), rabbit anti-Glial Fibrillary Acidic Protein (1:500; Dako, Rabbit polyclonal) and mouse anti- $\beta$ III tubulin (1:1000; Sigma, Tuj1), PGP9.5 antibody (1:500; UltraClone, UK), and p63 (1:200; Santa Cruz Biotechnology; H-137) followed by labeling with Alexa Fluor 488-conjugated donkey anti-goat IgG $(\mathrm{H}+\mathrm{L})$ (1:250; Molecular Probes, Eugene, OR) or Alexa Fluor 594-conjugated donkey anti-rabbit IgG $(\mathrm{H}+\mathrm{L})$ (1:250; Molecular Probes) or Alexa Fluor 555-conjugated donkey anti-rabbit IgG and Alexa Fluor 647-conjugated donkey anti-mouse IgG (1:500; Molecular Probes) along with Hoechst 33258 (10 mg/ml, Sigma) for nuclear staining. Images were collected with a confocal microscope system (LSM 510 META; Zeiss, Oberkochen, Germany). All mice were housed under specific pathogen-free conditions. All experimental procedures were approved by the Institutional Animal Care and Use Committee of Keio University.

\section{Results}

\section{SOX10 Expression was Clearly Delineated in Two} Distinct Subtypes of Human Salivary Gland Tumors

Among all 90 cases of the13 tumor types examined, SOX10 was frequently expressed in the cases of adenoid cystic carcinoma (22/23), acinic cell carcinoma (8/8), epithelial-myoepithelial carcinoma (5/5), carcinoma ex pleomorphic adenoma (10/10), myoepithelioma (4/4), and myoepithelial carcinoma (1/1). Acinic cell carcinoma cases, solid-type and one papillary-cystic type, were diffusely (>90\%) positive for SOX10 with high intensity but negative for other markers including p63 (Figure 1a-c). In Epithelial-myoepithelial carcinoma cases, SOX10 showed a biphasic expression pattern: a high proportion of both luminal and abluminal cells exhibited strong SOX10 expression (>90\%). On the other hand, p63 exhibited a monophasic expression pattern: positive for abluminal cells in a high $(>90 \%)$ proportion and high intensity, and completely negative in luminal cells (Figure 1d-f). As for tumors consisting of pure or predominant myoepithelial cells, such as myoepithelial carcino- ma, myoepithelioma and pleomorphic adenoma, the immunohistochemical profiles were similar. Calponin, Smooth muscle actin, S100, and p63 including SOX10 exhibited strong staining in over $70 \%$ of cells. All myoepithelial carcinoma $(n=1)$ and myoepithelioma $(n=4)$ cases consisted of spindle cells with various amounts of hyaline material, and SOX10 was positive in a high (>90\%) proportion and high intensity, the same as p63. In addition, the variable morphological types of pleomorphic adenoma, chondromyxoid, epithelioid, spindle, and plasmacytoid type, were positive for SOX10. Chondromyxoid type showed staining in $45 \%$ of cells with low intensity, epithelioid type in $75 \%$ with high intensity, spindle type in $>90 \%$ with high intensity, and plasmacytoid type in $>90 \%$ with high intensity. In a moderate number of carcinoma not otherwise specified cases, including carcinoma not otherwise specified components of carcinoma ex pleomorphic adenoma, SOX10 was positive $(5 / 22,23 \%)$. In adenoid cystic carcinoma samples exhibiting a typical cribriform structure, SOX10 stained $>80 \%$ luminal cells with high to low intensity and $>80 \%$ abluminal cells with high intensity, while p63 stained with high intensity only in abluminal cells (Figure 1g-i). Characteristically, the luminal cells of the 'biphasic true duct' exhibited SOX10 staining with high to low intensity, but did not exhibit staining with p63 and other markers. The abluminal cells of both the 'biphasic true duct' and 'pseudo-duct' structures showed $>70 \%$ positive staining for all markers examined. In one case of Adenoid cystic carcinoma exhibiting morphology with solid nests and minimal duct differentiation, SOX10 was expressed with high intensity in a high proportion ( $>90 \%$ ) of abluminal cells and with low focal intensity (40\%) in luminal cells. In contrast, p63 was expressed with high intensity in a large proportion ( $>90 \%$ ) of abluminal cells and was not expressed in luminal cells. Ten control samples (moderately to poorly differentiated squamous cell carcinoma; seven tongue and three oropharynx) were negative for SOX10 in carcinoma or background squamous epithelium. The immunohistochemical profiles of all markers tested for salivary gland tumors are shown in Table 1, and the SOX10 expression pattern is summarized in Table 2. Among all tumors, SOX10 tended to be expressed in both neoplastic myoepithelial cells and neoplastic epithelial cells, which mimicked acini and intercalated ducts.

\section{Histological Analysis of SOX10 and p63 Expression Patterns in the Human Adult Major Salivary Gland}

SOX10 was expressed in almost all (>90\%) of the abluminal (myoepithelial) and luminal (epithelial) cells in acini and intercalated ducts. SOX10 expression in mucinous acini was weaker than in serous acini. SOX10 was also consistently expressed 
a

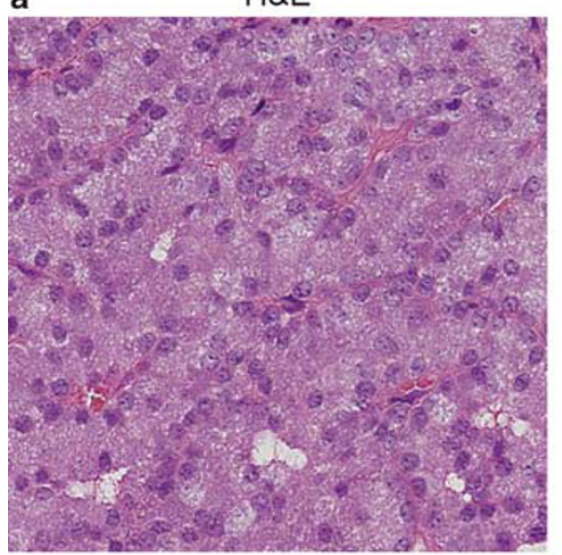

d

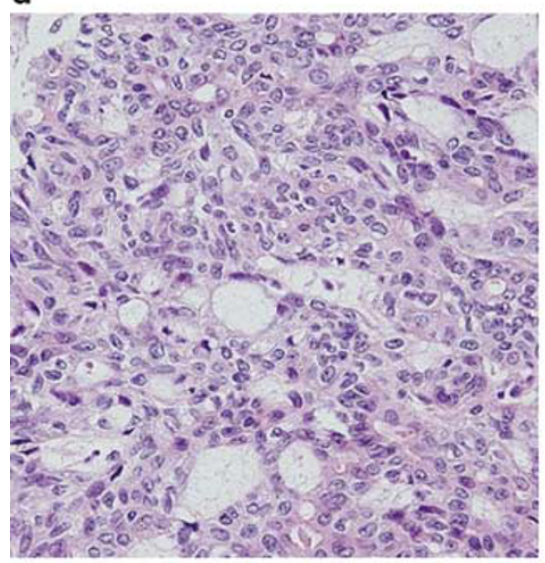

g

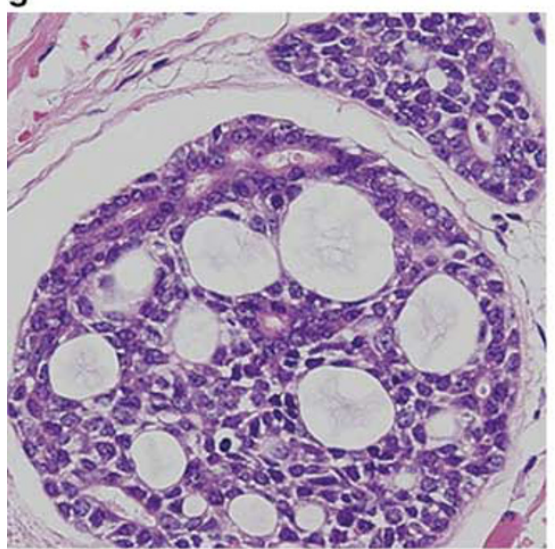

b

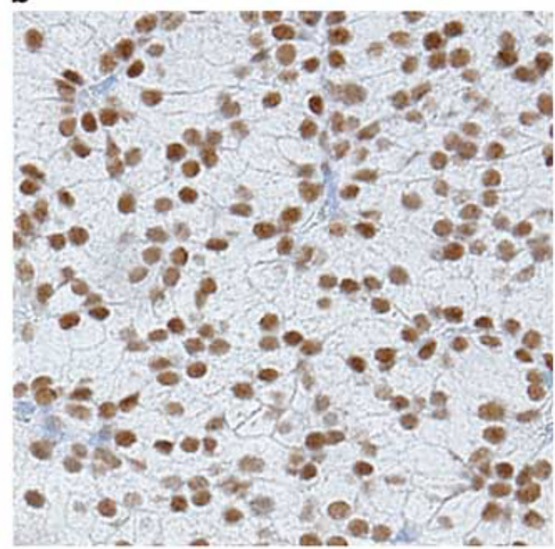

Acinic Cell Carcinoma

e

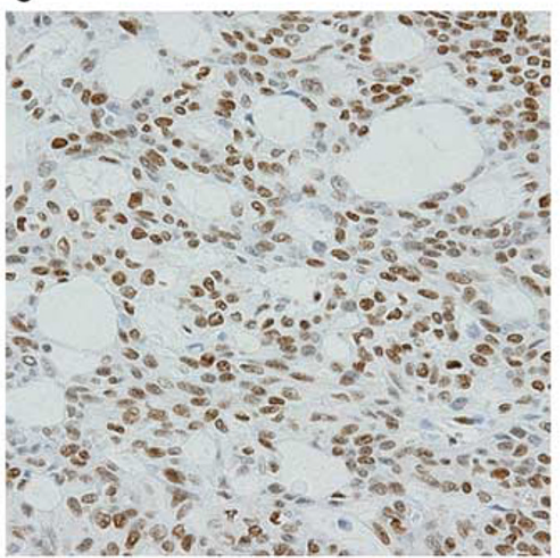

Epithelial-myoepithelial Carcinoma

h

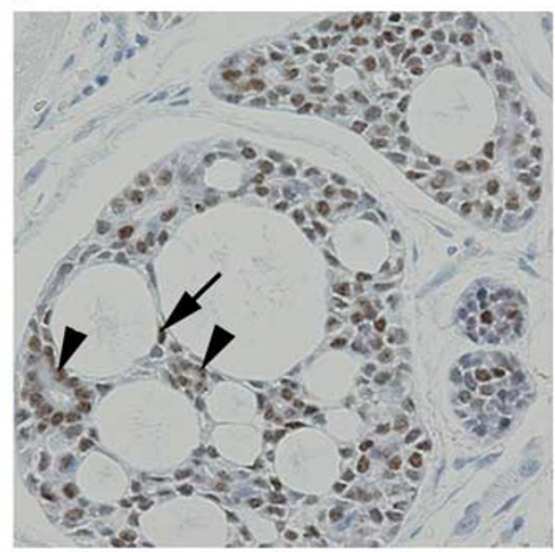

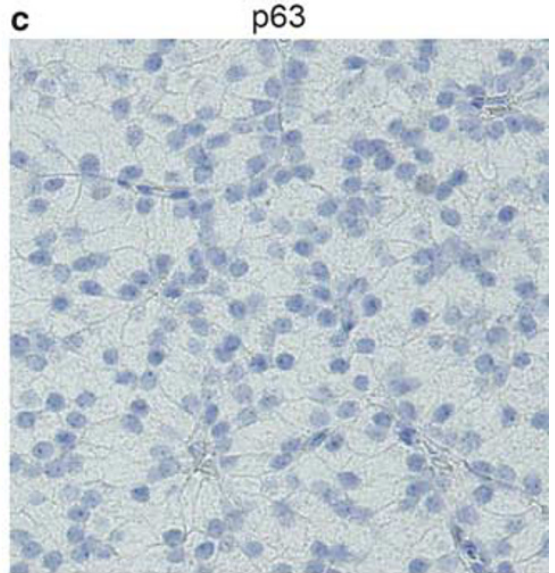

f

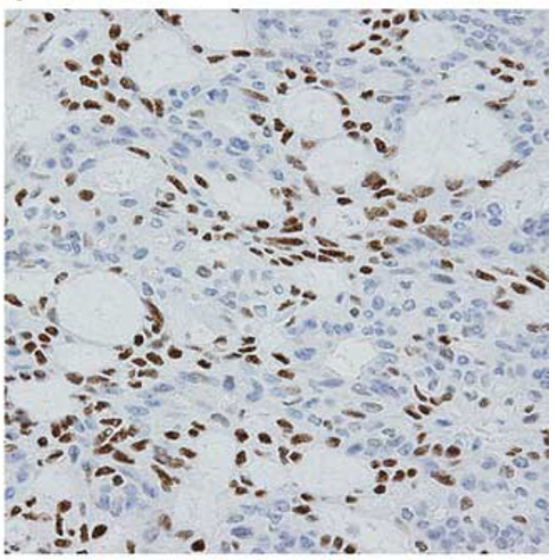

i

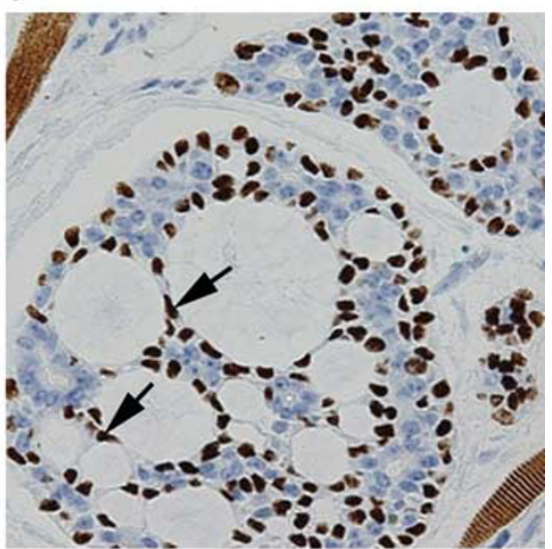

\section{Adenoid cystic carcinoma}

Figure 1 Expression patterns of SOX10 and p63 in acinic cell carcinoma, epithelial-myoepithelial carcinoma and adenoid cystic carcinoma. (a-c) A case of acinic cell carcinoma with a solid pattern. SOX10 is diffusely positive, and p63 is negative. (d-f) A case of epithelial-myoepithelial carcinoma. SOX10 exhibits a biphasic positive pattern of luminal and abluminal cells. Meanwhile, p63 is expressed only in abluminal cells. (g-i) A case of adenoid cystic carcinoma with a cribriform pattern. SOX10 is expressed both in luminal (arrow) and abluminal (arrowhead) cells of the true ducts (arrows). p63 is expressed in pesudoducts cells and abluminal cells of the true ducts (arrowhead). (a, d, g) H \& E staining; (b, e, h) SOX10; (c, f, i) p63.

in the nerves surrounding acini and ducts; however, SOX10 was not expressed in the abluminal (basal) and luminal cells of striated and excretory ducts. The intensity of SOX10 staining varied with location; while SOX10 showed high staining intensity in the luminal cells of serous acini and in the 
Table 1 Immunohistochemical profile of salivary gland tumors

\begin{tabular}{|c|c|c|c|c|c|c|c|}
\hline \multirow{2}{*}{ Histological diagnosis } & \multicolumn{7}{|c|}{ No. of positive cases (\%) } \\
\hline & $\mathrm{n}$ & SOX10 & p63 & $S M A$ & Calponin & $S 100$ & GFAP \\
\hline \multicolumn{8}{|l|}{ Malignant } \\
\hline ACC & 23 & $22(96)$ & 21 (91) & 21 (91) & $18(78)$ & 21 (91) & $5(21)$ \\
\hline AciCC & 8 & $8(100)$ & 0 & 0 & 0 & $1(13)$ & 0 \\
\hline SDC & 6 & 0 & 0 & 0 & 0 & $1(17)$ & $3(50)$ \\
\hline MuEC & 6 & 0 & $2(33)$ & 0 & 0 & $1(17)$ & $2(33)$ \\
\hline EMC & 5 & $5(100)$ & $5(100)$ & $5(100)$ & $3(60)$ & $4(80)$ & $3(60)$ \\
\hline MyEC & 1 & 1 & 1 & 1 & 0 & 0 & 0 \\
\hline OncCa & 1 & 0 & 0 & 0 & 0 & 0 & 0 \\
\hline CaNOS $^{a}$ & 22 & $5(23)$ & $10(45)$ & 0 & $4(18)$ & $9(41)$ & $5(23)$ \\
\hline \multicolumn{8}{|l|}{ Benign } \\
\hline $\mathrm{PA}^{\mathrm{b}}$ & 10 & $10(100)$ & $8(80)$ & $7(70)$ & $7(70)$ & $10(100)$ & $9(90)$ \\
\hline $\mathrm{ME}$ & 4 & $4(100)$ & $4(100)$ & $4(100)$ & $4(100)$ & $4(100)$ & $3(75)$ \\
\hline Onc & 2 & 0 & $2(100)$ & 0 & 0 & 0 & $1(50)$ \\
\hline WT & 2 & 0 & $2(100)$ & 0 & 0 & 0 & 0 \\
\hline
\end{tabular}

Abbreviations: ACC, adenoid cystic carcinoma; AciCC, acinic cell carcinoma; CaNOS, carcinoma not otherwise specified; EMC, epithelialmyoepithelial carcinoma; GFAP, glial fiburillary acidic protein; MuEC, mucoepidermoid carcinoma; MyEC, myoepithelial carcinoma; ME, myoepithelioma; Onc, Oncocytoma; OncCa, oncocytic carcinoma; PA, pleomorphic adenoma; SMA, smooth muscle actin; SDC, salivary duct carcinoma; WT, Warthin tumor.

When the case had $>10 \%$ positive cells, it was counted as positive.

ancluding CaNOS components of seven cases of carcinoma ex pleomorphic adenoma cases.

bIncluding PA components of four cases of carcinoma ex pleomorphic adenoma cases.

Table 2 Summary of SOX10 expression pattern in each salivary tumor and poorly differentiated squamous cell carcinoma

\begin{tabular}{|c|c|c|c|c|c|c|}
\hline $\begin{array}{l}\text { SOX10 } \\
\text { expression }\end{array}$ & & $\begin{array}{l}\text { Histologic } \\
\text { type }\end{array}$ & $\mathrm{n}$ & $\begin{array}{l}\text { No. of positive } \\
\text { cases }(\%)\end{array}$ & $\begin{array}{l}\text { Morphological pattern } \\
\text { of positive cases }\end{array}$ & $\begin{array}{l}\text { Proportion of positive cells } \\
\text { luminal/abluminal }\end{array}$ \\
\hline \multirow[t]{10}{*}{ Positive } & \multirow[t]{5}{*}{ Malignant } & ACC & 23 & $22(96)$ & $\begin{array}{l}\text { Biphasic duct (true duct) } \\
\text { Monophasic duct (pseudo duct) } \\
\text { Solid nest }\end{array}$ & \multirow{10}{*}{$\begin{array}{c}+1+++ \\
+++ \\
++ \\
+++ \\
++1+++ \\
+++ \\
+1++ \\
+ \\
+\end{array}$} \\
\hline & & AciCC & 8 & $8(100)$ & Sheet/cystic & \\
\hline & & EMC & 5 & $5(100)$ & Biphasic duct & \\
\hline & & MyEC & 1 & $1(100)$ & Spindle & \\
\hline & & CaNOS $^{a}$ & $22(7)$ & $5(23)$ & $\begin{array}{l}\text { Focal tubular formation } \\
\text { Solid nest }\end{array}$ & \\
\hline & \multirow[t]{5}{*}{ Benign } & $\mathrm{PA}^{\mathrm{b}}$ & $10(4)$ & $14(100)$ & Chondroid & \\
\hline & & & & & Epithelioid & \\
\hline & & & & & Spindle & \\
\hline & & & & & Plasmacytoid & \\
\hline & & $\mathrm{ME}$ & 4 & $4(100)$ & Spindle & \\
\hline \multirow{6}{*}{ Negative } & \multirow{3}{*}{ Malignant } & SDC & 6 & $0(0)$ & & \\
\hline & & MuEC & 6 & $0(0)$ & & \\
\hline & & OncCa & 1 & $0(0)$ & & \\
\hline & \multirow[t]{2}{*}{ Benign } & Onc & 2 & $0(0)$ & & \\
\hline & & WT & 2 & $0(0)$ & & \\
\hline & Malignant & SCC $^{\mathrm{C}}$ & 10 & $0(0)$ & & \\
\hline
\end{tabular}

Abbreviations: ACC, adenoid cystic carcinoma; AciCC, acinic cell carcinoma; EMC, epithelial-myoepithelial carcinoma; MyEC, myoepithelial carcinoma; CaNOS, carcinoma not otherwise specified; ME, myoepithelioma; MuEC, mucoepidermoid carcinoma; OncCa, oncocytic carcinoma; Onc, oncocytoma; PA, pleomorphic adenoma; SDC, salivary duct carcinoma; SCC, squamous cell carcinoma; WT, Warthin tumor.

,$+++>90 \%$ positive cells;,$++ 50-90 \% ;+, 10-50 \%$.

ancluding CaNOS components of seven cases of carcinoma ex pleomorphic adenoma cases.

bIncluding PA components of four cases of carcinoma ex pleomorphic adenoma cases.

${ }^{\mathrm{C}}$ Moderately to poorly differentiated squamous cell carcinoma of seven tongue and three oropharynx.

abluminal cells of acini and intercalated ducts, there was low intensity in the luminal cells of intercalated ducts. p63 was expressed in most ( $>90 \%)$ abluminal cells (both myoepithelial and basal cells) of acini, intercalated ducts, striated ducts, and excre- tory ducts, but not in luminal cells. The intensity of p63 did not differ among the abluminal cells. The difference in staining localization between SOX10-positive and p63-positive cells was apparent by immunofluorescence double staining, which 

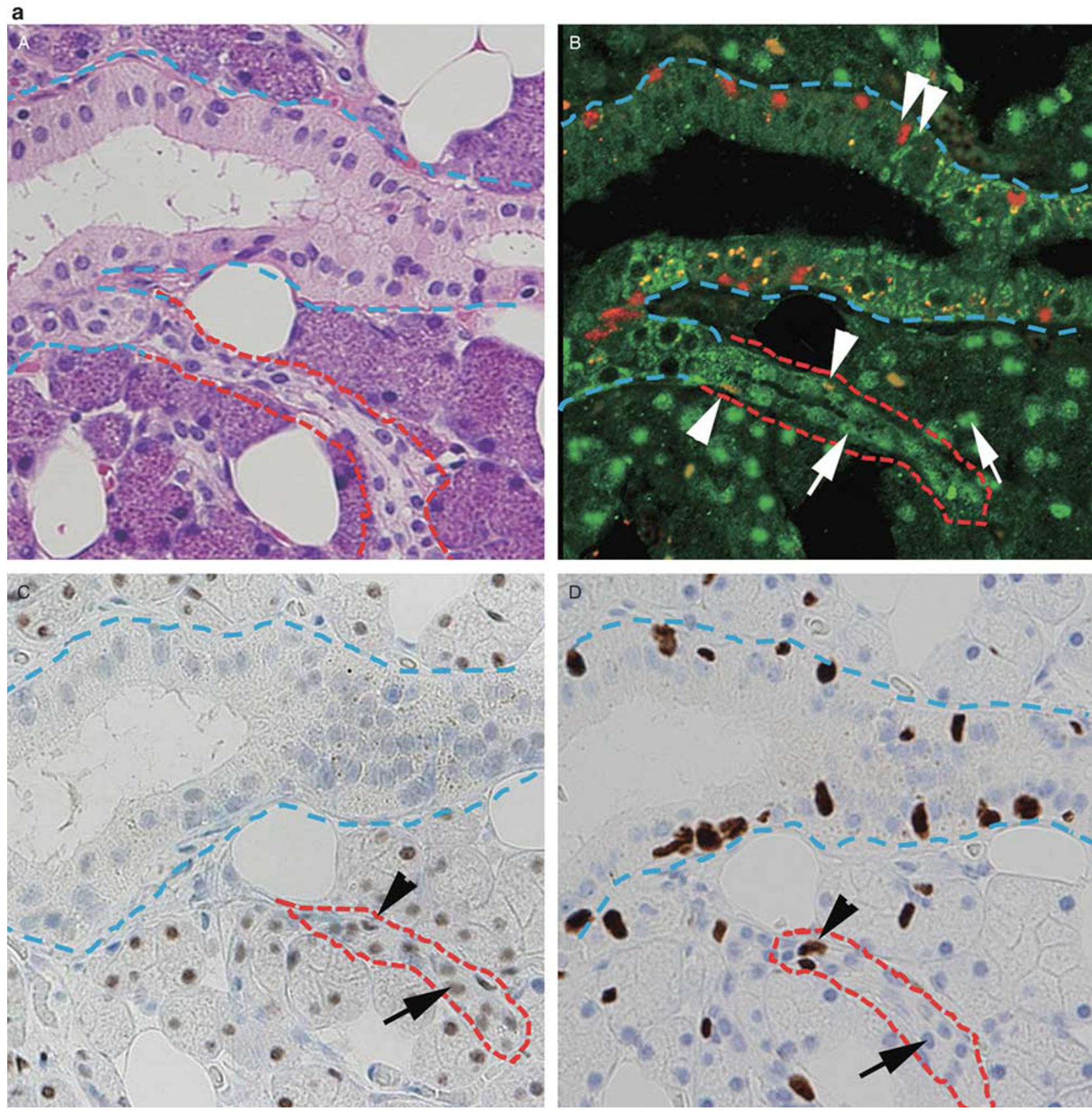

Figure 2 Presentation of SOX10, p63, and myoepithelial marker distribution in human normal salivary gland. (a) Expression patterns of SOX10 and p63 in the normal human salivary gland with immunohistochemistry. Serous acini, intercalated duct and striated duct of normal parotid glands. SOX10 expression is observed in luminal cells (arrow) of the intercalated duct (red dotted line), but not in luminal cells of the striated duct (blue dotted line). SOX10 and p63 are double-positive (yellow) in abluminal cells of the intercalated duct (arrowheads). Only p63 is observed in abluminal cells of the striated duct (double arrowheads). (A) H \& E, staining; (B) immunofluorescence double staining of SOX10 (green) and p63 (red); (C) SOX10; (D) p63. Schematic presentation of SOX10, p63, and myoepithelial marker distribution. (b) SOX10 was immunohistochemically positive in both luminal (epithelial) and abluminal (myoepithelial) cells of the serous acini and intercalated ducts; however, SOX10 was not expressed in the luminal cells of mucinous acini. p63 was expressed in all abluminal cells but not in luminal cells. Meanwhile, other markers except Glial fibrillary acidic protein were expressed only in normal myoepithelial cells examined.

showed that the luminal cells of acini and intercalated ducts were positive for SOX10, the abluminal (myoepithelial) cells of acini and intercalated ducts were double-positive for SOX10 and p63, the luminal cells of striated ducts and excretory ducts were negative for both, and the abluminal (basal) cells of striated ducts and excretory ducts were positive for p63 (Figure 2a). SOX10-positive tumors therefore exhibited morphological similarity to normal acini or intercalated ducts. 


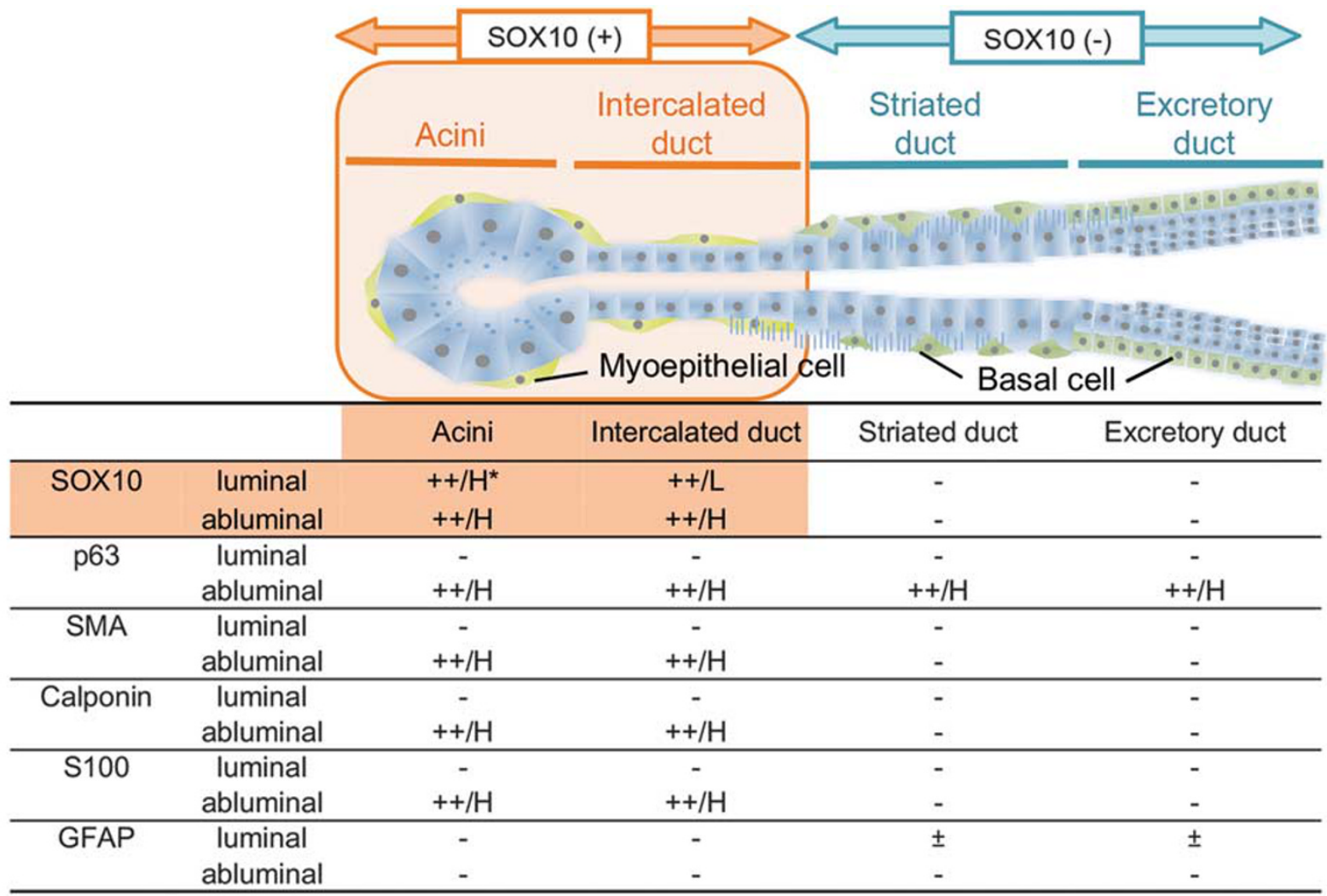

The proportion of positive cells :,$++>90 \%$ of positive cells;,$+ 11-90 \% ; \pm, 1-10 \%$; -, negative. The intensity of positive cells; $H$, high intensity; $L$, low intensity. ${ }^{*}$ Serous $(++)>$ Mucinous $(+)$ acini luminal cell SMA: Smooth muscle actin; GFAP: Glial fibrillary acidic protein

Figure 2 (Continued)

\section{Immunohistochemical Characteristics of the Human Major Salivary Gland SOX10, p63 and Other Myoepithelial Markers}

Smooth muscle actin and calponin were confirmed to be expressed in the cytoplasm of most ( $>90 \%$ ) of the myoepithelial cells of acini and intercalated ducts, but not in the basal cells of striated to excretory ducts or in the luminal cells of any segment. S100 was expressed at high intensity in every nerve and some $(>60 \%)$ of the myoepithelial cells of the acini and intercalated ducts, but not in either the basal cells of striated or excretory ducts, and not in the luminal cells of any segments. Glial fibrillary acidic protein was not expressed in abluminal cells of normal salivary glands or in the luminal cells of acini and intercalated ducts, but focal $(1-10 \%)$ positivity with high staining intensity was noted in the luminal cells of striated and excretory ducts. Thus, SOX10 was expressed in serous acini and intercalated ducts in human salivary glands. The immunohistochemical profiles of SOX10 and other markers are shown in luminal and abluminal cells from each segment of normal human salivary glands (Figure 2b).
Observing the Behavior of SOX10-Venus in the Major Salivary Gland from Developmental Stage to Adulthood

In SOX10-Venus mice at P0 stage, Venus + cells were clearly visualized in the parotid glands, submandibular glands and peripheral nerve fibers (Figure 3a). Microscopically, SOX10-Venus-positive cells were observed in the acini and the intercalated ducts but not in the striated ducts. In addition to the salivary glands, the surrounding spindle cells also expressed SOX10-Venus. These spindle cells were Schwann cells that closely associated with PGP9.5positive neural fibers (Figure $3 \mathrm{~b}$ ). We confirmed the colocalization of Venus fluorescence and endogenous SOX10 protein, which localized only in the nucleus. All SOX10-positive nuclei were labeled with Venus, which indicate that SOX10 expression was appropriately reported by Venus fluorescence in SOX10-Venus mouse strain (Figure 3c). As mentioned above, Glial fibrillary acidic protein was positive for striated and excretory ducts in the salivary gland. Also Glial fibrillary acidic protein is a well-known glial lineage cell marker, including Schwann cells. Tuj1 is also known as a neuronal 

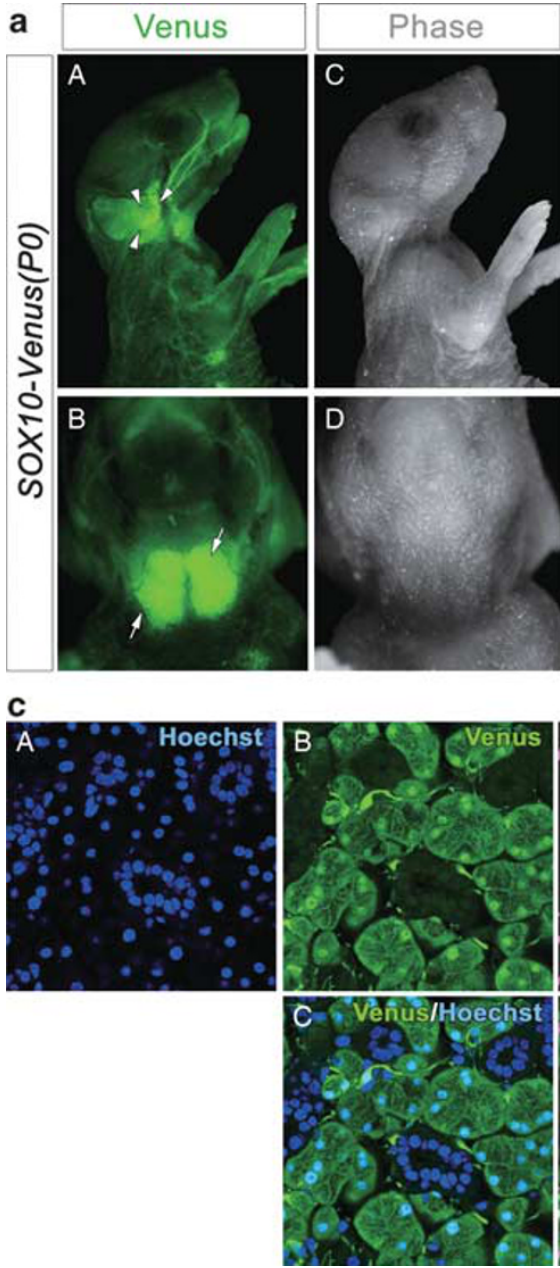
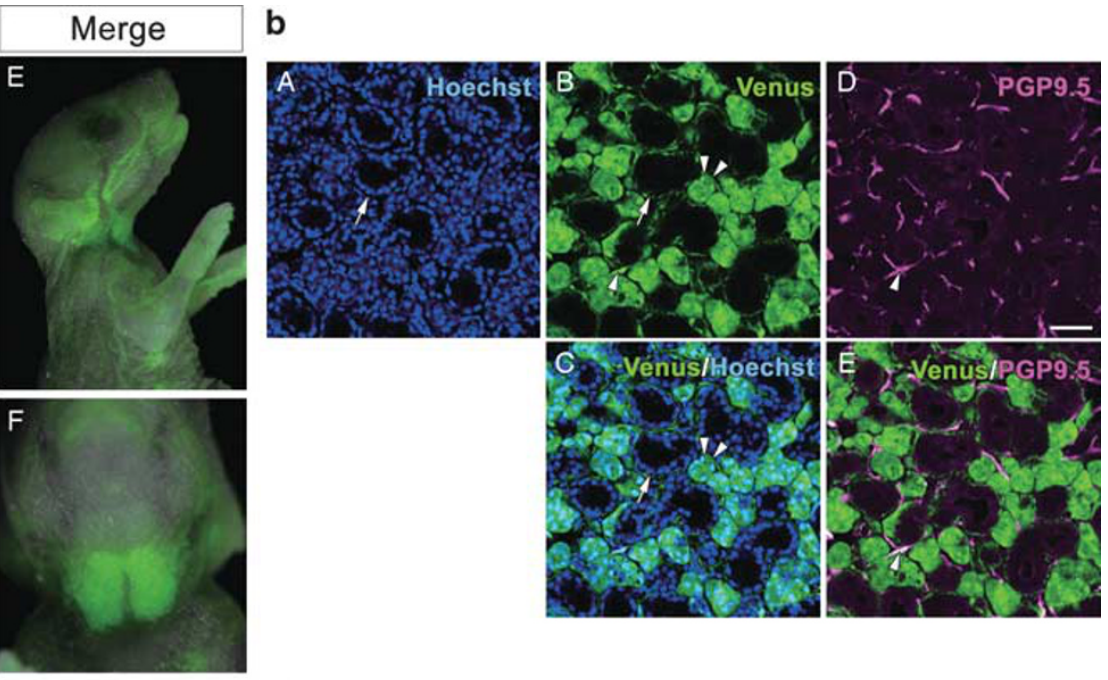

d
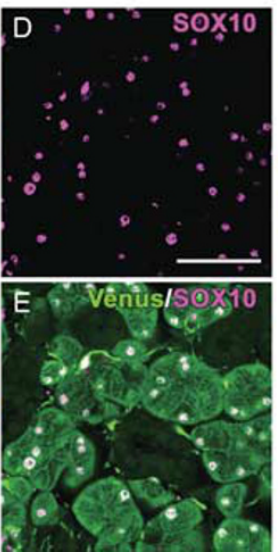
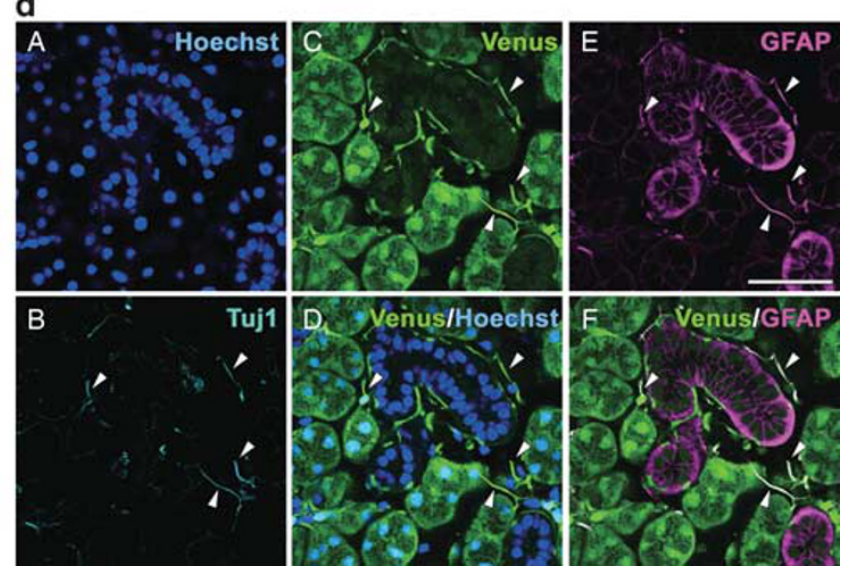

Figure 3 SOX10-Venus BAC transgenic mice exhibited SOX10-Venus in the major salivary glands in vivo. (a) Macroscopic views of SOX10-Venus mice. Upper panel: On P0 stage lateral view, the parotid glands (arrowheads) and peripheral nerve fibers were clearly visualized with Venus + cells. Lower panel: Front view of neck region, the submandibular glands (arrows) were clearly visualized with Venus + cells. (b) Microscopic views of SOX10-Venus mice submandibular gland. (A) Hoechst (nuclear stain); (B) Venus recognized with GFP antibody; (C) merged image of (A) and (B); (D) neuronal marker PGP9.5; (E) merged image of (B) and (D). The acini (double arrowheads) and neural fibers associated with Schwann cells (arrowheads) were Venus positive but negative in the striated ducts (arrows). (c) Colocalization of SOX10-Venus fluorescence and endogenous SOX10 protein. (A) Hoechst; (B) Venus recognized with GFP antibody; (C) merged image of (A) and (B); (D) SOX10 antibody; (E) merged image of (B) and (D). (d) SOX10-Venus showed a mutually exclusive pattern with Glial fibrillary acidic protein, except for Schwann cells (arrowheads). (A) Hoechst; (B) neuronal marker Tuj1 antibody; (C) Venus recognized with GFP antibody; (D) merged image of (A) and (B); (E) Glial fibrillary acidic protein (GFAP) antibody; (F) merged image of (C) and (E).

marker detected in neurites as well as the PGP9.5. SOX10-positive cells, which were labeled with Venus, showed a mutually exclusive pattern with Glial fibrillary acidic protein, except for Schwann cells, which localized adjacent to the neuronal marker Tuj1. These results indicated that the SOX10-positive cells consisted of three populations, acinic cells, intercalated ductal cells and Schwann cells. Among them, the only well-known neural crest derivatives were Schwann cells (Figure 3d). Thus, these findings suggest that, in mice, some major salivary gland cells may arise from the neural crest and continue to be expressed to adulthood. Histological analysis of the major salivary gland of SOX10-Venus mice revealed regions similar to human major salivary gland SOX10 expression patterns. Thus, SOX10 expression patterns imply that, in human major salivary glands, some acini, intercalated duct cells, and Schwann cells may arise from the SOX10-positive neural crest.

\section{Discussion}

In this study, we present the first comprehensive investigative report of SOX10 expression in human normal salivary glands and salivary gland tumors as well as mouse salivary gland development. We analyzed SOX10 expression in human samples of normal and neoplastic tissues and compared with several proteins that characterize and distinguish the various types of epithelial (luminal) and myoepithelial/basal (abluminal) cells of salivary glands by immunohistochemical staining. We showed that 
SOX10 was expressed in almost all acini and intercalated ducts of normal human major salivary glands in both luminal and abluminal cells. The biphasic expression pattern in acini and intercalated ducts contrasts with p63, which is expressed in abluminal cells, including both myoepithelial cells and basal cells, but never in luminal cells. In addition, the SOX10 expression pattern is different from those of Smooth muscle actin, calponin, S100 and Glial fibrillary acidic protein, which are often used as myoepithelial markers. Among these markers, only Glial fibrillary acidic protein was not expressed in normal myoepithelial cells, but was expressed in neoplastic myoepithelial cells, in agreement with previous data. ${ }^{12}$ Furthermore, CK5/6 and CK14 were expressed in the cytoplasm of most ( $>90 \%$ ) abluminal cells of acini to excretory ducts, and few $(<5 \%)$ of the luminal cells of excretory ducts (data not shown). The present results suggest that SOX10 is a useful marker to identify acini. This is also the first report in which SOX10 was frequently expressed in acinic cell carcinoma. Of our acinic cell carcinoma cases, of which seven were the solid-type and one case was the papillary-cystic type, were diffusely SOX10-positive. SOX10 was also positive in 5 of 22 cases of carcinoma ex pleomorphic adenoma. With regards to salivary gland tumors, our finding of SOX10 expression was unique and distinct from the expression profiles of other neoplastic myoepithelial markers, p63, smooth muscle actin, calponin, S100, and Glial fibrillary acidic protein. SOX10 was highly and frequently expressed in tumors exhibiting similarities to acini and intercalated ducts (eg, Acinic cell carcinoma, Adenoid cystic carcinoma, Pleomorphic adenoma, Epithelial-myoepithelial carcinoma, myoepithelioma, and myoepithelial carcinoma), and not in tumors resembling striated and excretory ducts (eg, mucoepidermoid carcinoma, salivary duct carcinoma, and warthin tumor). As a result, SOX10 expression pattern clearly delineated two distinct subtypes of human salivary gland tumors: acinic cell carcinoma, adenoid cystic carcinoma, epithelial-myoepithelial carcinoma, and myoepithelioma were SOX10 positive, whereas salivary duct carcinoma and mucoepidermoid carcinoma were SOX10 negative. Our data suggest that the survival of negative cases is significantly worse than positive cases. Numerous previous studies suggested that salivary duct carcinoma and mucoepidermoid carcinoma cases had a poor outcome compared with acinic cell carcinoma, adenoid cystic carcinoma, epithelial-myoepithelial carcinoma cases. ${ }^{13,14}$ Our overall survival data were also consistent with previous studies; however, the overall survival rate is only partially reliable as the cause of death and comorbidities were not taken into account.

This study builds upon past data ${ }^{2}$ that established SOX10 as a marker of myoepithelial tumors other than Schwann and melanocytic tumors, and confirmed SOX10 negativity in neoplastic epithelial cells of multiple organs; lung, breast, esophagus, stomach, colon, liver, kidney, prostate gland, uterine cervix, ovary, and skin. Our findings are not limited to neoplastic myoepithelial cells but include some neoplastic epithelial cells, which are commensurate with SOX10 distribution in the normal salivary glands examined. DOG1 has been reported as a marker of salivary acinus and intercalated duct differentiation; ${ }^{15}$ however, the immunohistochemical profile of SOX10 is different from DOG1. SOX10 is diffusely positive for normal myoepithelial cells, which are reported to be completely negative for DOG1. Therefore, we can emphasize that the SOX10 expression pattern of salivary gland tumors mirrors those of normal tissues, showing acinus and intercalated duct differentiation in a biphasic manner. Myoepithelial cells are known to show various morphologies, chondromyxoid, spindle, epithelioid and plasmacytoid, and SOX10 is positive in all of these cells. The immunohistochemical profiles of nonneoplastic and neoplastic myoepithelial cells have been documented in several studies. ${ }^{12,16,17}$ Consistent with these reports, S100 and Glial fibrillary acidic protein, classical myoepithelial cell markers, which are similar to SOX10, exhibit low sensitivity and specificity for myoepithelial cells. SOX10 sensitivity to myoepithelial cells most closely resembles Smooth muscle actin and calponin, which are often used as sensitive and specific markers. These results indicated that a subgroup of carcinoma not otherwise specified has differentiation toward acini or intercalated ducts, and may be derived from acini or intercalated ducts. Some reports have indicated that intercalated duct hyperplasia and adenoma might be precursor lesions to epithelial-myoepithelial carcinoma or other biphasic tumors. ${ }^{18}$ It is also known that intercalated ducts exhibit high proliferation activity under conditions of postradiation and chronic sialadenitis. ${ }^{19}$ Intercalated ducts are considered to have high potential for entering the neoplastic process, and several tumors might have originated from intercalated ducts. ${ }^{18,20}$ Elucidating tumor lineage leads to understanding of the complicated salivary gland tumor morphology. SOX10 can offer clues for establishing the histogenesis and spectrum. To understand SOX10 expression and salivary gland histogenesis, we employed SOX10-Venus mice. These mice are useful tools for studying neural crest-derived organs in the pathological process. ${ }^{7}$ SOX10-Venus clearly exhibited not only neural cells but also major salivary glands and lacrimal glands. SOX10 was present at all stages of salivary gland morphogenesis from the initial bud to terminal stage in the SOX10-Venus mouse experimental study (data not shown). The mice SOX10-Venus observation showed similarity to human salivary gland development (Figure 3). These results indicate 
that major salivary glands likely derive from SOX10-positive cells arising in the neural crest. Recently, Shakhova et $a l^{21}$ showed that SOX10 silencing in human melanoma cells suppresses neural crest stem cell properties. The human adult major salivary gland shows persistent SOX10 and p63 double-positive cells in the acini and intercalated ducts in the abluminal cells. The coexpression of SOX10 and p63 in salivary tumors may explain the striking range of morphologic diversity in salivary tumors.

In summary, SOX10 expression clearly delineates two distinct subtypes of human salivary gland tumors and its clinical and biological significance was clarified: acinic cell carcinoma, adenoid cystic carcinoma, epithelial-myoepithelial carcinoma, and myoepithelioma were SOX10 positive, whereas salivary duct carcinoma and mucoepidermoid carcinoma were SOX10 negative. These results suggest that acinic cell carcinoma, adenoid cystic carcinoma, Epithelial-myoepithelial carcinoma, and myoepithelioma have acinar or intercalated duct origins. Thus, SOX10 can aid in diagnosing and understanding the histogenesis of salivary gland tumors.

\section{Acknowledgements}

We thank Dr Chad Tang for advice and very helpful comments on this manuscript. We are grateful to Ms Sachiko Miura, Ms Chizu Kina, and Ms Tomoko Umaki for skilled technical assistance. This work was supported in part by a Grant-in-Aid for Scientific Research (C) to TM and a Grant-in-Aid for Young Scientists (B) to RO from the Ministry of Education, Culture, Sports, Science, and Technology (MEXT) of Japan.

\section{Disclosure/conflict of interest}

The authors declare no conflict of interest.

\section{References}

1 Kelsh RN. Sorting out SOX10 functions in neural crest development. Bioessays 2006;28:788-798.

2 Nonaka D, Chiriboga L, Rubin BP. SOX10: a panschwannian and melanocytic marker. Am J Surg Pathol 2008;32:1291-1298.

3 Roh J, Cho EA, Seong I, et al. Down-regulation of SOX10 with specific small interfering RNA promotes transdifferentiation of Schwannoma cells into myofibroblasts. Differentiation 2006;74:542-551.

4 Knox SM, Hoffman MP. Salivary gland development and regeneration, In: Wong DT(eds). Salivary Diagnostics. Wiley-Blackwell: Ames, 2008, pp 3-13.

5 Lombaert IMA, Hoffman MP. Epithelial stem/progenitor cells in the embryonic mouse submandibular gland, In: Tucker AS, Miletich I (eds). Salivary Glands; Development, Adaptation and Disease. Vol. 14 Karger: Basel, 2010, pp 90-106.
6 Deal KK, Cantrell VA, Chandler RL, et al. Distant regulatory elements in a SOX10-beta GEO BAC transgene are required for expression of SOX10 in the enteric nervous system and other neural crest-derived tissues. Dev Dyn 2006;235:1413-1432.

7 Eveson JW, Auclair P, Gnepp DR, et al. World Health Organization Classification of Tumours, Pathology and Genetics of Head and Neck Tumours, Chapter 5. Tumors of the salivary glands. IARC Press: Lyon, 2005, pp 209-215.

8 Yamamoto S, Tsuda $\mathrm{H}$, Honda $\mathrm{K}$, et al. Actinin-4 expression in ovarian cancer: a novel prognostic indicator independent of clinical stage and histological type. Mod Pathol 2007;20:1278-1285.

9 Effendi K, Mori T, Komuta M, et al. Bmi-1 gene is upregulated in early-stage hepatocellular carcinoma and correlates with ATP-binding cassette transporter B1 expression. Cancer Sci 2010;101:666-672.

10 Mori T, Kiyono T, Imabayashi $\mathrm{H}$, et al. Combination of hTERT and bmi-1, E6, or E7 induces prolongation of the life span of bone marrow stromal cells from an elderly donor without affecting their neurogenic potential. Mol Cell Biol 2005;25:5183-5195.

11 Shibata S, Yasuda A, Renault-Mihara F, et al. SOX10Venus mice: a new tool for real-time labeling of neural crest lineage cells and oligodendrocytes. Mol Brain 2010;3:31.

12 Ianez RF, Buim ME, Coutinho-Camillo CM, et al. Human salivary gland morphogenesis: myoepithelial cell maturation assessed by immunohistochemical markers. Histopathology 2010;57:410-417.

13 Dardick I. Color Atlas/Text of Salivary Gland Tumor Pathology. Igaku-shoin medical Publishers: New York; 1996, pp 7-16.

14 Gillespie MB, Albergotti WG, Eisele DW. Recurrent salivary gland cancer. Curr Treat Options Oncol 2012;13:58-70.

15 Chênevert J, Duvvuri U, Chiosea S, et al. DOG1: a novel marker of salivary acinar and intercalated duct differentiation. Mod Pathol 2012;25:919-929.

16 Prasad AR, Savera AT, Gown AM, et al. The myoepithelial immunophenotype in 135 benign and malignant salivary gland tumors other than pleomorphic adenoma. Arch Pathol Lab Med 1999;123: 801-806.

17 Okura M, Hiranuma T, Tominaga G, et al. Expression of S-100 protein and glial fibrillary acidic protein in cultured submandibular gland epithelial cells and salivary gland tissues. Histogenetic implication for salivary gland tumors. Am J Pathol 1996;148:1709-1716.

18 Weinreb I, Seethala RR, Hunt JL, et al. Intercalated duct lesions of salivary gland: a morphologic spectrum from hyperplasia to adenoma. Am J Surg Pathol 2009;33:1322-1329.

19 Burlage FR, Faber H, Kampinga HH, et al. Coppes RP.Enhanced proliferation of acinar and progenitor cells by prophylactic pilocarpine treatment underlies the observed amelioration of radiation injury to parotid glands. Radiother Oncol 2009;90:253-256.

20 Chetty R. Intercalated duct hyperplasia: possible relationship to epithelial-myoepithelial carcinoma and hybrid tumours of salivary gland. Histopathology 2000;37:260-263.

21 Shakhova O, Zingg D, Schaefer SM, et al. SOX10 promotes the formation and maintenance of giant congenital naevi and melanoma. Nat Cell Biol 2012; 14:882-890. 